\title{
Base-Frequency Negative Sequence Impedance Modeling and Sensitivity Analysis of DFIG
}

\author{
Simin PANG ${ }^{\mathrm{a}, 1}$, Ruiyuan JIA ${ }^{\mathrm{a}}$, Qihui LIU ${ }^{\mathrm{a}}$, Linlin $\mathrm{WU}^{\mathrm{b}}$ and Hui LIU ${ }^{\mathrm{b}}$ \\ ${ }^{a}$ Department of Electrical and Electronic Engineering, University of North China \\ Electric Power, Beijing, China \\ ${ }^{\mathrm{b}}$ State Grid Jibei Electric Co. Ltd. Research Institute, Beijing, China
}

\begin{abstract}
The phenomenon of three phase voltage imbalance frequently occurs in large-scale new energy grid connected areas in China; in severe cases, a large number of wind turbines will be disconnected from the grid. To solve the problem of the voltage imbalance at the point of common coupling (PCC), analyze the influence of generator parameters change on negative sequence voltage under the background of unbalanced power grid, a modeling method of base-frequency negative sequence impedance of doubly fed induction generator (DFIG) which including phase locked loop (PLL), rotor side converter (RSC) and grid side converter (GSC) is proposed. By establishing the negative sequence equivalent circuit of grid-connected system of DFIG, the relationship between the negative sequence voltage of PCC and the negative sequence impedance of DFIG is listed, and analyzing the sensitivity of control parameters link to base-frequency impedance, the parameter that has great influence on base-frequency negative sequence impedance of PCC is found out. Finally, the accuracy of impedance modeling and sensitivity analysis is verified by simulation studies.
\end{abstract}

Keywords. Doubly fed induction generator, new energy grid, voltage imbalance

\section{Introduction}

With the continuous development of China's new energy industry, wind power generation has occupied an important position in the power industry. However, due to the increasing capacity of wind farms, the areas where large-scale wind power converges are characterized by weak grid structure, lack of reactive power compensation devices and small local load, which are prone to voltage imbalance $[1,2]$.

At present, the research on the dominant factors that cause the voltage imbalance in the wind power collection area mainly focuses on the three-phase load imbalance, threephase line parameters are not equal, and three-phase line without transposition, etc. The unbalanced load is the main factor that causes the unbalanced three-phase voltage in the region is proposed in Ref. [3]. Ref. [4] shows that due to the imbalance of the outgoing circuit, the positive and negative sequence coupling exists between the circuits, thus generating negative sequence voltage through interaction. The influence of transformer

${ }^{1}$ Corresponding Author, Simin PANG, Department of Electrical and Electronic Engineering, University of North China Electric Power, Beijing, China; Email: 476673746@qq.com. 
insulation damage on the voltage imbalance at the wind turbine outlet is analyzed in Ref. [5]. However, the factors causing voltage imbalance in the above literatures are mainly caused by equipment other than the wind turbine, and the influence of negative sequence impedance characteristics of the wind turbine itself on voltage imbalance is not considered. In the case of unbalanced load, the negative sequence impedance model of three-phase off-grid inverter is established in Ref. [6]. By exploring the relationship between harmonic voltage and current at the outlet side of the DFIG, the harmonic equivalent model of the wind turbine is obtained in Refs. [7-8]. All the above literatures have established impedance models at frequencies other than the base-frequency, but there are few studies on the base-frequency negative sequence impedance models of DFIG.

Firstly, the base-frequency negative sequence impedance model of DFIG is established in this paper, which considering the RSC, GSC and PLL structures. Secondly, the impedance model is combined with sensitivity analysis to analyze the influence of wind turbine control parameters on the base-frequency negative sequence voltage at PCC. Finally, the sensitivity analysis results correctness is verified by the MATLAB timedomain simulation results.

\section{Base-Frequency Negative Sequence Impedance Modeling of DFIG}

\subsection{Basic Circuit of the DFIG Grid-Connected System}

The grid-connected system of the DFIG is shown in figure 1. It is assumed that there are base-frequency negative sequence voltage and current at the PCC, the wind turbine can be equivalent to a controlled DFIG system and a GSC system by ignoring the voltage fluctuation of the DC bus, the controller dead zone and the switching frequency of power electronic devices [9].

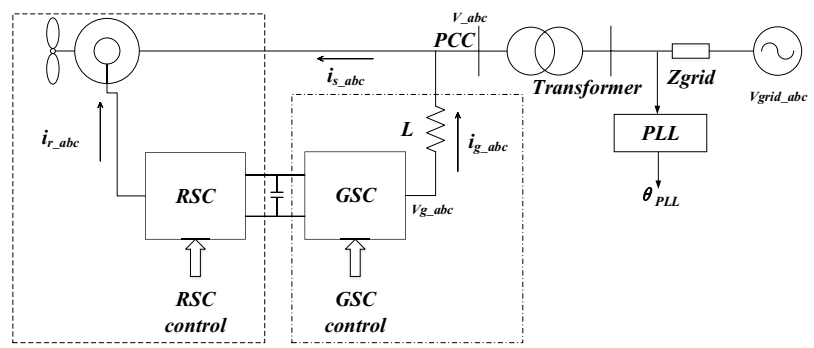

Figure 1. Grid-connected system of DFIG.

According to figure 1, the relation between the output current and voltage of GSC and the voltage of PCC is shown in equation (1).

$$
\left[\begin{array}{ccc}
L & & \\
& L & \\
& & L
\end{array}\right] \cdot \frac{d}{d t}\left[\begin{array}{c}
i_{g a} \\
i_{g b} \\
i_{g c}
\end{array}\right]=\left[\begin{array}{c}
v_{g a} \\
v_{g b} \\
v_{g c}
\end{array}\right]-\left[\begin{array}{c}
v_{a} \\
v_{b} \\
v_{c}
\end{array}\right]
$$


In the equation, $i_{\mathrm{g}_{a} \text { abc }}$ and $v_{\mathrm{g}_{\_} \mathrm{abc}}$ are the three-phase current and voltage at the GSC side, $v_{-a b c}$ is the three-phase voltage at PCC, and $\mathrm{L}$ is the filtering inductance.

Using symmetric component method to transform equation (1), the positive, negative and zero-order components of voltage and current are obtained.

$$
\frac{1}{3}\left[\begin{array}{ccc}
3 L & & \\
& 3 L & \\
& & 3 L
\end{array}\right] \cdot \frac{d}{d t}\left[\begin{array}{c}
i_{g 1} \\
i_{g 2} \\
i_{g 0}
\end{array}\right]=\left[\begin{array}{c}
v_{g 1} \\
v_{g 2} \\
v_{g 0}
\end{array}\right]-\left[\begin{array}{c}
v_{1} \\
v_{2} \\
v_{0}
\end{array}\right]
$$

In the equation, subscripts 1,2 and 0 represent positive, negative and zero-order components respectively.

The expressions of $v_{a}$ and $i_{g a}$ in time domain are shown in equations (3) and (4).

$$
\begin{aligned}
& v_{a}(t)=V_{1} \cos \left(\omega_{1} t+\varphi_{v 1}\right)+V_{n} \cos \left(\omega_{1} t+\varphi_{v n}\right) \\
& i_{g a}(t)=I_{1} \cos \left(\omega_{1} t+\varphi_{i 1}\right)+I_{n} \cos \left(\omega_{1} t+\varphi_{i n}\right)
\end{aligned}
$$

In the equation, $V_{1}, I_{1}, V_{n}, I_{n}$ are the voltage and current amplitudes of base-frequency positive and negative sequence waves which at the PCC respectively, $\varphi_{v l}, \varphi_{i l}, \varphi_{v n}$ and $\varphi_{i n}$ are the initial phase angles of the base-frequency positive and negative sequence waves respectively, $\omega_{1}$ is the fundamental angular frequency.

For simple calculation, equations (3) and (4) are converted into the frequency domain to obtain equations (5) and (6).

$$
\begin{gathered}
\boldsymbol{V}_{a}[f]=\left\{\begin{array}{l}
\boldsymbol{V}_{1}, f= \pm f_{1} \\
\boldsymbol{V}_{n}, f= \pm f_{1}
\end{array}\right. \\
\boldsymbol{I}_{g a}[f]=\left\{\begin{array}{l}
\boldsymbol{I}_{1}, f= \pm f_{1} \\
\boldsymbol{I}_{n}, f= \pm f_{1}
\end{array}\right.
\end{gathered}
$$

In the equation, $\boldsymbol{V}_{1}=\left(V_{1} / 2\right) e^{ \pm j \varphi_{v 1}}, \varphi_{v 1}=0$ is the initial phase of the fundamental voltage.

The expressions of voltage and current in the frequency domain of the other two phases can be obtained by using the same method described above.

\subsection{PLL Output Angle}

Figure 2 is a structure block diagram of the PLL. The voltage at PCC is controlled by the PLL to obtain a transformation angle $\theta_{P L L}$ that contains the base-frequency negative sequence component perturbation $\triangle \theta$. 


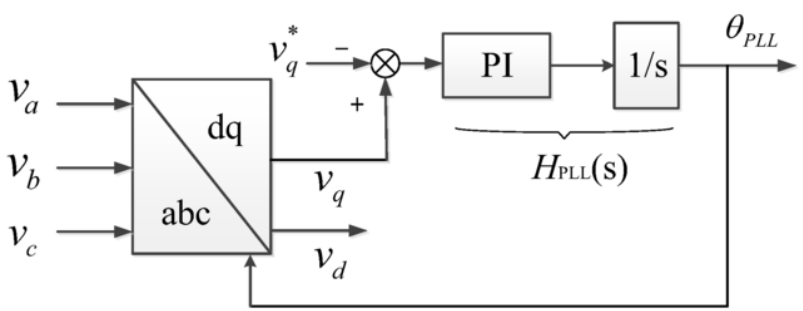

Figure 2. PLL structure block diagram.

The frequency domain expression of the base-frequency negative sequence voltage corresponding to the disturbance component $\Delta \theta$ is shown in equation (7).

$$
\begin{gathered}
\Delta \theta[f]= \pm j F(s) \boldsymbol{V}_{n}, f= \pm 2 f_{1} \\
F(s)=\frac{H_{P L L}(s)}{1+V_{1} H_{P L L}(s)}
\end{gathered}
$$

In the equation, $H_{P L L}(s)=\left(K_{p p}+K_{p i} / \mathrm{s}\right) / \mathrm{s}$ is the transfer function of the PLL PI regulator. $K_{p p}$ and $K_{p i}$ are PLL PI regulator ratio and integral parameters respectively.

Using the idea of PLL harmonic linearization, the relation between the output phase angle of PLL and the control link is analyzed, and the coordinate transformation matrix $\mathrm{T}\left(\theta_{P L L}\right)$ containing the disturbance of the base-frequency negative sequence component is obtained.

$$
T\left(\theta_{P L L}\right)=\frac{2}{3}\left[\begin{array}{lll}
\cos \theta_{P L L} & \cos \left(\theta_{P L L}-2 \pi / 3\right) & \cos \left(\theta_{P L L}+2 \pi / 3\right) \\
-\sin \theta_{P L L} & -\sin \left(\theta_{P L L}-2 \pi / 3\right) & -\sin \left(\theta_{P L L}+2 \pi / 3\right)
\end{array}\right]
$$

In the above equation,

$$
\cos \theta_{P L L}[f]= \begin{cases}\frac{1}{2}, & f= \pm f_{1} \\ \frac{1}{2} F\left(s \pm j 2 \pi f_{1}\right) \boldsymbol{V}_{n}, & f= \pm f_{1} \\ -\frac{1}{2} F\left(s \mp j 2 \pi f_{1}\right) \boldsymbol{V}_{n}, f= \pm 3 f_{1}\end{cases}
$$

The other elements are no longer listed individually.

\subsection{Base-Frequency Negative Sequence Impedance Modeling of GSC System}

Ignoring the influence of the GSC voltage outer loop and considering the disturbance influence of the current regulator and the base-frequency negative sequence voltage, the relationship between base-frequency negative sequence voltage and current at GSC side 
is analyzed. Finally, the base-frequency negative sequence impedance of the GSC system can be obtained.

The three phase current at the AC side of GSC is transformed by the transformation matrix (9) to obtain the dq axis component of the AC side current of the GSC.

$$
\begin{gathered}
\boldsymbol{I}_{g d}[f]= \begin{cases}I_{1} \cos \varphi_{i 1}, & f=0 \\
\boldsymbol{I}_{n} \pm j \sin \varphi_{i 1} I_{1} F(s) \boldsymbol{V}_{n}, & f= \pm 2 f_{1}\end{cases} \\
\boldsymbol{I}_{g q}[f]= \begin{cases}I_{1} \sin \varphi_{i 1}, & f=0 \\
\pm j \boldsymbol{I}_{n} \mp j \cos \varphi_{i 1} I_{1} F(s) \boldsymbol{V}_{n}, & f= \pm 2 f_{1}\end{cases}
\end{gathered}
$$

In the actual operation control system of the wind turbine, the control links are all carried out in the dq coordinate system, therefore, the GSC current regulator control block diagram is shown in figure 3 . In this figure, $i_{g d}$ and $i_{g q}$ are the actual values of the $\mathrm{d}$ and $\mathrm{q}$ axis currents; $i_{g d_{-} r e f}$ and $i_{g q_{-} r e f}$ are the reference values of the $\mathrm{d}$ and $\mathrm{q}$ axis currents; $v_{g d_{-} r e f}$ and $v_{g q_{-} r e f}$ are the voltage reference values of $\mathrm{d}$ and $\mathrm{q}$ axis output by the converter; $H_{g i}(s)$ is the transfer function of PI regulator; $K_{d q}=\omega_{1} L$ is the GSC current loop coupling coefficient.

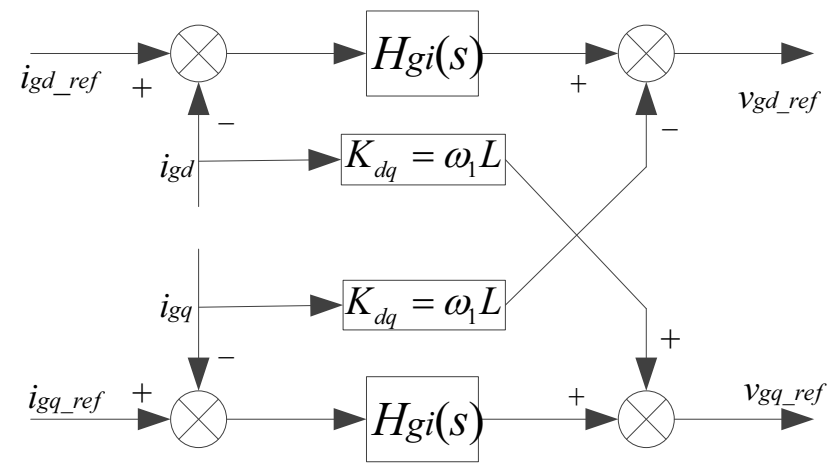

Figure 3. GSC current regulator control block diagram.

When the system is adjusted to a steady state, the base-frequency positive sequence current can stably follow the current reference value, while for the base-frequency negative sequence current, its current reference value is 0. Therefore, the GSC side current containing the base-frequency positive and negative sequence components passes through the current regulator, and the $\mathrm{d}$ and $\mathrm{q}$ axis voltage reference values output by the converter are obtained as shown in equations (13) and (14).

$$
\boldsymbol{V}_{g d_{\_} r e f}[f]= \begin{cases}-K_{d q} I_{1} \sin \varphi_{i 1}+D_{g 0}, & f=0 \\ {\left[-H_{g i}(s) \mp j K_{d q}\right] \boldsymbol{I}_{n}+j I_{1} F(s) \boldsymbol{V}_{n}\left(\mp \sin \varphi_{i 1} H_{g i}(s) \pm \cos \varphi_{i 1} K_{d q}\right), f= \pm 2 f_{1}}\end{cases}
$$




$$
\boldsymbol{V}_{g q_{-} r e f}[f]=\left\{\begin{array}{lc}
K_{d q} I_{1} \cos \varphi_{i 1}+Q_{g 0}, & f=0 \\
{\left[\mp j H_{g i}(s)+K_{d q}\right] \boldsymbol{I}_{n}+j I_{1} F(s) \boldsymbol{V}_{n}\left( \pm \cos \varphi_{i 1} H_{g i}(s) \pm \sin \varphi_{i 1} K_{d q}\right), f= \pm 2 f_{1}}
\end{array}\right.
$$

In the equation, $\mathrm{D}_{g 0}$ and $\mathrm{Q}_{g 0}$ are $\mathrm{DC}$ steady state indicators of GSC output, $H_{g i}(s)=K_{g p}+K_{g i} / \mathrm{s}$ is the transfer function of PI regulator, $K_{g p}$ and $K_{g i}$ are the proportion and integral coefficient of PI regulator respectively.

By inversely transforming the above two expressions to the $a b c$ coordinate system, substituting the reference voltage value of a-phase into the frequency domain expression of equation (1), the expressions of $\mathrm{D}_{g 0}, \mathrm{Q}_{g 0}$ can be obtained as shown in equation (15).

$$
\left\{\begin{array}{l}
D_{0}=-2 \pi f_{1} L I_{1} \sin \varphi_{i 1}+K_{d q} I_{1} \sin \varphi_{i 1}+V_{1} \\
Q_{0}=2 \pi f_{1} L I_{1} \cos \varphi_{i 1}-K_{d q} I_{1} \cos \varphi_{i 1}
\end{array}\right.
$$

Substituting the second part of equation (15) into the frequency domain expression of equation (2), the relationship between the voltage and the current of base-frequency negative sequence of the GSC system is summarized as shown in equation (16).

$$
\left(-H_{g i}\left(s \pm j \omega_{1}\right) \mp j K_{d q}\right) \boldsymbol{I}_{n}+F\left(s \pm j \omega_{1}\right) \boldsymbol{V}_{n}\left(\boldsymbol{I}_{1}^{*} H_{g i}\left(s \pm j \omega_{1}\right)+\frac{D_{0}}{2} \mp j \frac{Q_{0}}{2}\right)-\boldsymbol{V}_{n}=L \cdot s \cdot \boldsymbol{I}_{n}
$$

According to the direction of current and voltage in the calculation circuit, the basefrequency negative sequence impedance of GSC system can be arranged as follows.

$$
Z_{g s c}^{n}=-\frac{\boldsymbol{V}_{n}}{I_{n}}=\frac{H_{g i}(2 s)+j K_{d q}+j \omega_{1} L}{1-F(2 s)\left[\boldsymbol{I}_{1}^{*} H_{g i}(2 s)+\frac{D_{0}}{2}-j \frac{Q_{0}}{2}\right]}
$$

\subsection{Modeling of Base-Frequency Negative Sequence Impedance of Controlled DFIG} System

In the DFIG system, the rotor component converted to the dq coordinate system is more than the stator component by the rotor position angle $\theta_{r}$. Therefore, the reference angle of coordinate transformation of the controlled DFIG system is $\theta_{P L L}-\theta_{r}$. The coordinate transformation matrix $T\left(\theta_{P L L}-\theta_{r}\right)$ is

$$
T\left(\theta_{P L L}-\theta_{r}\right)=\frac{2}{3}\left[\begin{array}{lll}
\cos \left(\theta_{P L L}-\theta_{r}\right) & \cos \left(\theta_{P L L}-\theta_{r}-2 \pi / 3\right) & \cos \left(\theta_{P L L}-\theta_{r}+2 \pi / 3\right) \\
-\left(\sin \theta_{P L L}-\theta_{r}\right) & -\sin \left(\theta_{P L L}-\theta_{r}-2 \pi / 3\right) & -\sin \left(\theta_{P L L}-\theta_{r}+2 \pi / 3\right)
\end{array}\right]
$$

In the above equation, 


$$
\cos \left(\theta_{P L L}-\theta_{r}\right)[f]=\left\{\begin{array}{l}
\frac{1}{2}, \quad f= \pm\left(f_{1}-f_{r}\right) \\
\frac{1}{2} F\left(s \pm j 2 \pi f_{s}\right) \boldsymbol{V}_{n}, f= \pm\left(f_{1}+f_{r}\right) \\
-\frac{1}{2} F\left(s \mp j 2 \pi f_{s}\right) \boldsymbol{V}_{n}, f= \pm\left(3 f_{1}-f_{r}\right)
\end{array}\right.
$$

The other elements are no longer listed individually.

The control block diagram of the RSC current regulator is shown in figure 4 . In this figure: $H_{r i}(s)=K_{r p}+K_{r i} / \mathrm{s}$ is the transfer function of PI regulator in RSC current loop, $K_{\mathrm{rp}}$ and $K_{\mathrm{ri}}$ are respectively the proportion and integral coefficient of PI regulator; $K_{r d q}=\omega_{s}\left(L_{r}-L_{m}{ }^{2} / L_{s}\right)$ is the decoupling coefficient of RSC current loop, $\omega_{\mathrm{s}}=\omega_{1}-\omega_{\mathrm{r}} ; L_{r}$ is self-induction of rotor, $L_{m}$ is mutual inductance of stator and rotor, $L_{s}$ is the self-induction of stator. $i_{r d}$ and $i_{r q}$ are the rotor actual current values of $\mathrm{d}$ and $\mathrm{q}$ axis; $i_{r d_{-} \text {ref }}$ and $i_{r q}{ }_{r e f}$ are the rotor reference current value of $\mathrm{d}$ and $\mathrm{q}$ axis; $v_{r d_{-} r e f}$ and $v_{r q_{-}}$ref are the $\mathrm{d}$ and $\mathrm{q}$ axis voltage command values output by RSC.

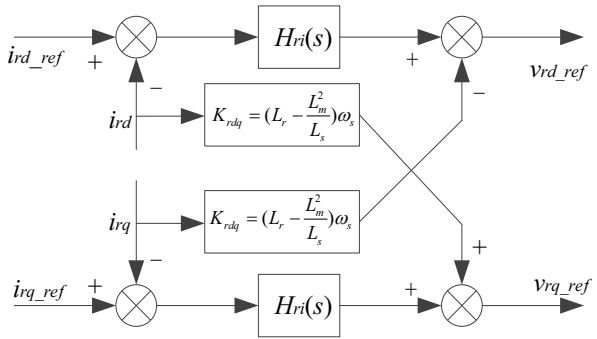

Figure 4. Control block diagram of RSC current regulator.

According to the same method described in Section 2.3, the base-frequency negative sequence impedance model of the controlled DFIG system is sorted out and obtained, as shown in equation (20).

$$
Z^{n}{ }_{r}=\frac{\mathrm{V}_{n}}{\mathrm{I}_{s n}}=\frac{R_{s}+j \omega_{1} L_{s}-\frac{s^{2} L_{m}^{2} \rho_{n}(s)}{R_{r}+s L_{r} \rho_{n}(s)+\left[H_{r i}(2 s)+j K_{r d q}\right] K_{e}^{2}}}{1-\frac{s L_{m} F(2 s) K_{e}\left\{\mathrm{I}_{r 1}\left[H_{r i}(2 s)\right]+\frac{D_{r 0}}{2}-j \frac{Q_{r 0}}{2}\right\}}{R_{r}+s L_{r} \rho_{n}(s)+\left[H_{r i}(2 s)+j K_{r d q}\right] K_{e}^{2}}}
$$

In the above formula,

$$
\left\{\begin{array}{c}
D_{r 0}=\left[R_{r}+\frac{\omega_{s} \omega_{1} L_{m}^{2} R_{s}}{R_{s}^{2}+\left(\omega_{1} L_{s}\right)^{2}}\right] \frac{I_{r d}}{K_{e}^{2}}+\left[\frac{\omega_{s} \omega_{1}^{2} L_{m}^{2} L_{s}}{R_{s}^{2}+\left(\omega_{1} L_{s}\right)^{2}}-\omega_{s} L_{r}\right] \frac{I_{r q}}{K_{e}^{2}}+\frac{\omega_{s} \omega_{1} L_{m} L_{s}}{R_{s}^{2}+\left(\omega_{1} L_{s}\right)^{2}} \frac{V_{s d}}{K_{e}}-\frac{\omega_{s} L_{m} R_{s}}{R_{s}^{2}+\left(\omega_{1} L_{s}\right)^{2}} \frac{V_{s q}}{K_{e}}+I_{r q} K_{r d q} \\
Q_{r 0}=\left[R_{r}+\frac{\omega_{s} \omega_{1} L_{m}^{2} R_{s}}{R_{s}^{2}+\left(\omega_{1} L_{s}\right)^{2}}\right] \frac{I_{r q}}{K_{e}^{2}}+\left[\omega_{s} L_{r}-\frac{\omega_{s} \omega_{1}^{2} L_{m}^{2} L_{s}}{R_{s}^{2}+\left(\omega_{1} L_{s}\right)^{2}}\right] \frac{I_{r d}}{K_{e}^{2}}+\frac{\omega_{s} \omega_{1} L_{m} L_{s}}{R_{s}^{2}+\left(\omega_{1} L_{s}\right)^{2}} \frac{V_{s q}}{K_{e}}+\frac{\omega_{s} L_{m} R_{s}}{R_{s}^{2}+\left(\omega_{1} L_{s}\right)^{2}} \frac{V_{s d}}{K_{e}}-I_{r d} K_{r d q} \\
\rho_{n}(s)=\frac{s+j \omega_{r}}{s}
\end{array}\right.
$$




\section{Base-Frequency Negative Sequence Impedance Model and Verification of Grid- Connected DFIG System}

\subsection{Base-Frequency Negative Sequence Impedance Model of DFIG Grid-Connected System}

According to the circuit structure of the grid-connected DFIG system, by connecting the base-frequency negative sequence impedance of GSC system and controlled DFIG system in parallel, the expression of base-frequency negative sequence impedance of DFIG system can be obtained as shown in formula (23).

$$
Z_{D F I G}^{n}=\frac{Z_{g s c}^{n} \cdot Z_{r}^{n}}{Z_{r}^{n}+Z_{g s c}^{n}}
$$

According to the DFIG base-frequency negative sequence impedance, the basefrequency negative sequence equivalent circuit of DFIG grid-connected system is shown in figure 5. In this figure, $Z^{n}$ grid is the base-frequency negative sequence equivalent impedance of the power grid and its lines, $i_{g n}$ is the base-frequency negative sequence current of PCC, $u_{g n}$ is the power grid base-frequency negative sequence voltage, $v_{p c c}^{n}$ is the base-frequency negative sequence voltage of PCC.

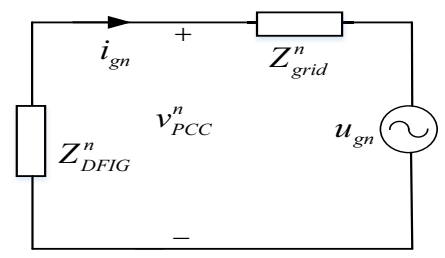

Figure 5. DFIG grid-connected system negative sequence equivalent circuit diagram.

According to figure $5, v_{p c c}^{n}$ is related to the negative sequence impedance of wind turbine, and its expression is as follows:

$$
v_{P C C}^{n}=\frac{u_{g n} Z_{D F I G}^{n}}{Z_{\text {grid }}^{n}+Z_{D F I G}^{n}}
$$

It can be seen from the equation (24) that the greater the base-frequency negative sequence impedance of DFIG system, the greater the base-frequency negative sequence voltage at PCC.

\subsection{Verification of Base-Frequency Negative Sequence Impedance Model}

In order to verify the accuracy of the base-frequency negative sequence impedance modeling proposed in this paper, the time domain simulation model of the gridconnected DFIG system is built based on the MATLAB software as shown in figure 1, 
and the model parameters are shown in table 1.

Table 1. Main parameters of model.

\begin{tabular}{llllllll}
\hline Parament & Value & Parament & Value & Parament & Value & Parament & Value \\
\hline$R_{s}$ & 0.023 p.u. & $L_{m}$ & 2.9 p.u. & $K_{r i}$ & 8 & $U_{d c}$ & $1150 \mathrm{~V}$ \\
$R_{r}$ & 0.016 p.u. & $K_{g p}$ & 0.83 & $K_{p p}$ & 5 & $U_{s}$ & $575 \mathrm{~V}$ \\
$L_{r}$ & 3.06 p.u. & $K_{g i}$ & 5 & $K_{p i}$ & 30 & $K_{e}$ & 0.291 \\
$L_{s}$ & 3.08 p.u. & $K_{r p}$ & 0.6 & $P$ & $1.5 \mathrm{MW}$ & $f$ & $60 \mathrm{~Hz}$ \\
\hline
\end{tabular}

In the time domain simulation model, the voltage imbalance of the power grid is set as $3 \%$, and the wind speed is changed to make the DFIG operate under different working conditions. The measured base-frequency negative sequence impedance at the wind turbine exit is compared with the calculated results of the analytical formula, as shown in table 2. It can be seen from table 2 that the error level between the time domain simulation results and the analytical formula calculation results is $10^{-3}$. The error is small, which verifies the accuracy of the analytical formula.

Table 2. Comparison between analytical formula and simulation results.

\begin{tabular}{llll}
\hline Wind speed & Formula calculation results & Time domain simulation results & Error magnitude \\
\hline $6 \mathrm{~m} / \mathrm{s}$ & $0.02838+0.09374 \mathrm{i} \Omega$ & $0.02725+0.09058 \mathrm{i} \Omega$ & $10^{-3}$ \\
$8 \mathrm{~m} / \mathrm{s}$ & $0.02459+0.07577 \mathrm{i} \Omega$ & $0.02327+0.07483 \mathrm{i} \Omega$ & $10^{-3}$ \\
\hline $10 \mathrm{~m} / \mathrm{s}$ & $0.02035+0.06231 \mathrm{i} \Omega$ & $0.01954+0.06167 \mathrm{i} \Omega$ & $10^{-3}$ \\
$12 \mathrm{~m} / \mathrm{s}$ & $0.01839+0.05862 \mathrm{i} \Omega$ & $0.01797+0.05680 \mathrm{i} \Omega$ & $10^{-3}$ \\
$14 \mathrm{~m} / \mathrm{s}$ & $0.01839+0.05862 \mathrm{i} \Omega$ & $0.01791+0.05689 \mathrm{i} \Omega$ & $10^{-3}$ \\
$16 \mathrm{~m} / \mathrm{s}$ & $0.01839+0.05862 \mathrm{i} \Omega$ & $0.01791+0.05689 \mathrm{i} \Omega$ & $10^{-3}$ \\
\hline
\end{tabular}

\section{Sensitivity Analysis and Verification of Base-Frequency Negative Sequence Impedance}

\subsection{Sensitivity Analysis of Base-Frequency Negative Sequence Impedance}

According to section 3.1, the base-frequency negative sequence voltage at PCC is positively correlated with the DFIG base-frequency negative sequence impedance. Therefore, by analyzing the sensitivity of each control parameter of wind turbine to basefrequency negative sequence impedance, the relationship between parameters and voltage at PCC can be obtained.

The base-frequency negative sequence impedance sensitivity [10] of DFIG is defined as

$$
K=\frac{\partial Z_{D F I G}^{n}}{\partial m}
$$

In the above equation: $\mathrm{m}$ represents the wind turbine control parameters to be analyzed for sensitivity.

The control parameters considered in this paper are as follows: the proportional parameter $K_{p p}$ and the integral parameter $K_{p i}$ of the PLL PI regulator; GSC current loop proportional parameter $K_{g p}$ and integral parameter $K_{g i}$; RSC current loop proportional parameter $K_{r p}$ and integral parameter $K_{r i}$. 
According to the impedance model and sensitivity definition described above, the parameter value is set at $0-2$ p.u. The sensitivity curves of the real and imaginary parts of the base-frequency negative sequence impedance and the control parameters of wind turbine are drawn as shown in figure 6.

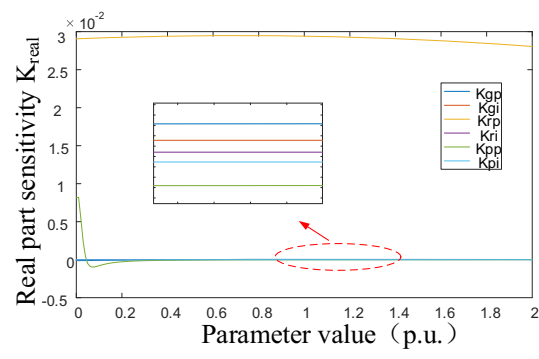

(a)

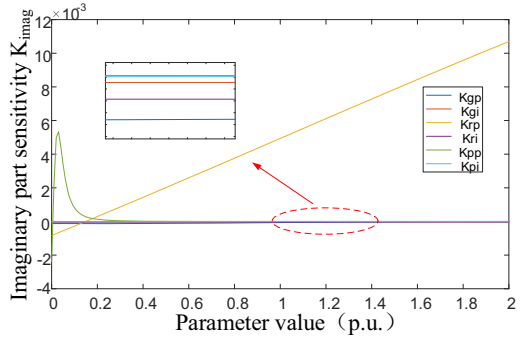

(b)

Figure 6. Real (resistance) and imaginary (reactance) parts sensitivity of base-frequency negative sequence impedance.

Figure 6a shows the sensitivity of real part(resistance) of impedance. It can be seen from the figure that the sensitivity of real part of $K_{r p}$ is much greater than other parameters, and the real part of base-frequency negative sequence impedance increases with the increase of $K_{r p}$; Other parameters have little impedance sensitivity and have little influence on impedance.

Figure $6 \mathrm{~b}$ shows the sensitivity of imaginary part(reactance) of impedance. It can be seen from the figure that the sensitivity of imaginary part of $K_{r p}$ is greater than other parameters when the parameters are in the range of $0.2-2 \mathrm{p} . \mathrm{u}$, and the imaginary part of base-frequency negative sequence impedance increases with the increase of $K_{r p}$, while the impedance sensitivity of other parameters is very small and has little influence on impedance.

\subsection{Sensitivity Analysis and Verification of Control Parameters}

To verify the correctness of the above theoretical analysis, a time-domain simulation model is built in MATLAB/Simulink, and the grid voltage unbalance degree is set to $5 \%$. The values of control parameters are changed at $5 \mathrm{~s}$, and the waveform diagram of basefrequency negative sequence voltage at PCC is shown in figure 7.

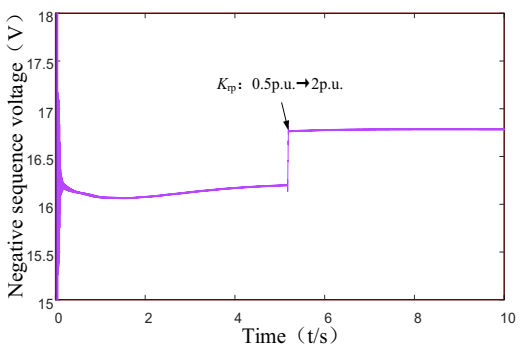

(a)

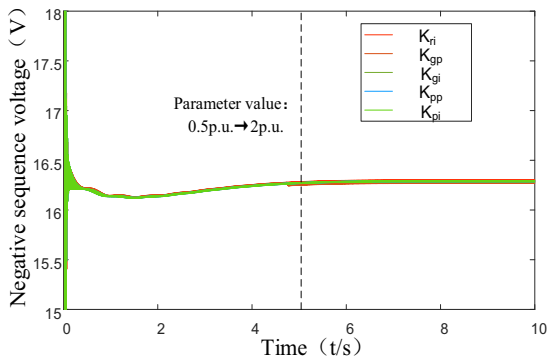

(b)

Figure 7. Negative sequence voltage at PCC when changing control parameter values. 
It can be seen from figure 7 that changing the parameter of $K_{r p}$ has the greatest impact on the base-frequency negative sequence voltage of PCC, while changing other parameters has almost no effect on the negative sequence voltage of $\mathrm{PCC}$, which verifies the accuracy of the above sensitivity analysis.

\section{Conclusion}

Aiming at the problem of voltage imbalance at PCC, this paper proposes a DFIG basefrequency negative sequence impedance modeling method, and verifies the correctness of the impedance model through time domain simulation; At the same time, this paper analyzes the impedance sensitivity of each control parameter of the wind turbine, and obtains the conclusion that the RSC current loop proportional parameter $K_{r p}$ has the greatest influence on the base-frequency negative sequence impedance of the wind turbine.

\section{Acknowledgments}

This paper is supported by the project "Voltage quality control technology in power grids with large-scale renewables" (4000-202014051A-0-0-00).

\section{References}

[1] Chen C, Liu H, Wu L L, et al. 2019 Voltage unbalance mechanism research with large-scale wind power integration 2019 IEEE Innovative Smart Grid Technologies 1608-12.

[2] Xu D K 2018 Research on the Control Scheme to Damping DFIG-Associated SSR on the Grid Side in Guyuan Wind Farms (North China Electric Power University).

[3] Zhang B, Jing M C and Zhang J H 2010 Utilizing the static var compensator for solving wind electric field three-phase unbalance problems Mechanical \&. Electrical Engineering Technology 39 (3) 99-103.

[4] Liu H, Chen C, Ju Y T, et al. 2020 Characteristics analysis and suppression strategy of voltage unbalance in areas with integration of wind power Automation of Electric Power Systems 1-11.

[5] Styliaras N 2016 Investigation of the Effect of the Transformer Connection Type on Voltage Unbalance Propagation: Case Study at Nasudden Wind Farm (Uppsala, Sweden: Uppsala University).

[6] Luo J, Zhang H Y, Zhang S, et al. 2020 Modeling and characteristic analysis of sequential impedance of three-phase off-grid inverter under unbalanced load Power System Protection and Control 48 (12) 3340.

[7] Nian H and Yang H Y 2016 Impedance modeling and stability analysis of grid-connected inverter under unbalanced operation conditions Automation of Electric Power Systems 40 (10) 76-83.

[8] Shen Y W, Wang S W, Zhang B, et al. 2020 Harmonic modeling and characteristic analysis of converters in doubly fed wind power generation Proceedings of the CSU-EPSA 32 (9) 105-112.

[9] Yang H Y 2016 Impedance Modeling and Stability Analysis of Doubly Fed Induction Generator System (Zhejiang University).

[10] Ju P, Guo L, Gao C P, et al. 2010 Frequency-domain sensitivities with application to power system modeling Proceedings of the Chinese Society for Electrical Engineering 30 (28) 19-24. 